\title{
UM PANORAMA DA DISCUSSÃO SOBRE ANSIEDADE NOS PERIÓDICOS NACIONAIS DE ANÁLISE DO COMPORTAMENTO
}

\author{
AN ANALYTICAL-BEHAVIORAL OVERVIEW ON THE DISCUSSIONS OF ANXIETY \\ IN NATIONAL JOURNALS
}

\begin{abstract}
Letícia de Paula Von Backschat ${ }^{1}$, Carolina Laurenti ${ }^{*}$
${ }^{1}$ Graduanda do curso de Psicologia da Universidade Estadual de Maringá.

${ }^{2}$ Doutora em Filosofia pela Universidade Federal de São Carlos, Professora adjunta do Departamento de Psicologia da Universidade Estadual de Maringá.

*Avenida Colombo, 5790 - Jardim Universitário - CEP: 87020-900 - Maringá-PR. Universidade Estadual de Maringá. Departamento de Psicologia (bloco 118, sala 04). E-mail: claurenti@uem.br
\end{abstract}

Submetido em: 30/12/2019; Aceito em: 02/03/2020.

\section{RESUMO}

Segundo a Organização Mundial de Saúde, em 2017, o Brasil foi considerado o país mais ansioso do mundo, com 9,3\% da população diagnosticada com 0 transtorno. Explicar e tratar a ansiedade torna-se, então, um desafio para diferentes teorias psicológicas, dentre elas a Análise do Comportamento. $O$ objetivo deste artigo é sistematizar como a literatura analítico-comportamental nacional tem discutido o comportamento ansioso. Para isso, foi realizada uma pesquisa bibliográfico-exploratória, cujas fontes foram artigos publicados em revistas nacionais especializadas em Análise do Comportamento, e que apresentaram algum dos subtipos de ansiedade tipificados pelo DSM-V. Foram selecionados e documentados 15 artigos, cujas informações foram agrupadas em categorias de análise que compilaram as principais temáticas associadas à discussão da ansiedade. A literatura analítico-comportamental critica concepções teleológicas e internalistas de ansiedade, e a entende como um fenômeno comportamental complexo, uma vez que sua explicação pode envolver aspectos filogenéticos, ontogenéticos e culturais. A despeito de sua complexidade, as discussões analítico-comportamentais se concentraram no nível ontogenético. Nesse domínio, o comportamento ansioso exibe interações de respostas reflexas e operantes, com topografias abertas e encobertas, que ocorrem com a função de evitar o contato com estímulos aversivos condicionados. Embora possa também ser entendido como "normal", foram citados diferentes modelos e técnicas psicoterápicas analítico-comportamentais (FAP, SiMCCIT) para lidar com o comportamento ansioso como queixa clínica. Destaca-se a importância de a Análise do Comportamento expandir os estudos sobre a ansiedade ao nível cultural, buscando estratégias de prevenção e enfrentamento do fenômeno com potencial de alcance mais amplo.

Palavras-chave: Ansiedade. Literatura analítico-comportamental. Psicopatologia. Análise do comportamento. 


\section{ABSTRACT}

According to the World Health Organization, in 2017, Brazil was considered the most anxious country in the world, with $9.3 \%$ of the population diagnosed with the disorder. Thus, explaining and treating anxiety have become a challenge for different psychological theories, including Behavior Analysis. The aim of this paper is to systematize how the national analytical-behavioral literature has discussed anxious behavior. For this, a bibliographic-exploratory research has been conducted, whose sources are articles published in national journals specialized in Behavior Analysis, and which presented some of the anxiety subtypes used by the DSM-V. Fifteen articles have been selected and documented, whose information has been grouped into analysis categories compiling the main themes associated with the discussion of anxiety. The analytical-behavioral literature criticizes teleological and internalist conceptions of anxiety, and understands it as a complex behavioral phenomenon, since its explanation involves phylogenetic, ontogenetic and cultural aspects. Despite its complexity, analytical-behavioral discussions have focused on the ontogenetic level. In this domain, anxious behavior exhibits interactions of reflex and operant responses, with overt and covert topographies, which occur in order to avoid contact with conditioned aversive stimuli. Although it can also be understood as "ordinary", different models and analytical-behavioral psychotherapeutic techniques (FAP, SiMCCIT) have been cited to deal with anxious behavior as a clinical complaint. It highlights the importance of Behavior Analysis to expand the studies on anxiety at the cultural level, seeking strategies for prevention and coping with the phenomenon with wider potentiality.

Keywords: Anxiety. Analytical-behavioral. Psychopathology. Behavior Analysis.

\section{INTRODUÇÃO}

A World Health Organization (WHO, ou OMS em português) divulgou em 2017 um relatório que continha as estimativas globais de depressão e outras doenças mentais consideradas mais comuns. Nesse material, foi indicado que o Brasil era o país mais ansioso do mundo: 9 em cada 100 brasileiros tinham o diagnóstico de ansiedade (9,3\% da população), proporção três vezes maior que a média mundial de 4 em cada 100 indivíduos (3,6\%). Esse valor correspondia a aproximadamente 20 milhões de brasileiros, em sua maioria mulheres, uma vez que da população feminina total $7,7 \%$ receberam 0 diagnóstico, enquanto nos homens a incidência foi de 3,6\%. O relatório apontava, ainda, que houve um aumento de 15\% nos casos de ansiedade entre 2005 e 2015 (WHO, 2017).

Esses dados desafiam diferentes áreas do conhecimento a explicar e a contribuir para o tratamento e prevenção desse transtorno. Dentre elas, destaca-se a Psicologia e, em particular, a Análise do Comportamento, uma proposta de psicologia científica pautada na filosofia do Comportamentalismo Radical. De acordo com essa filosofia, os fenômenos psicológicos são entendidos em termos de comportamento, que é elucidado olhando-se para a 
relação inextrincável entre 0 indivíduo e o mundo social e não-social (SKINNER, 2003).

O comportamento humano é considerado um fenômeno complexo, já que emerge da conjunção de três histórias: a filogenética, que compreende a história evolutiva da espécie; a ontogenética, que abrange a história de vida do indivíduo; e a história da cultura da qual o indivíduo faz parte (PRADO, 2013). É no nível filogenético que são constituídas as características anatomofisiológicas, e comportamentos típicos da espécie, como, por exemplo, o reflexo incondicionado (SKINNER, 2006). Já o nível cultural demonstra que o comportamento humano também se constitui no bojo de contingências sociais organizadas por grupos e que são transmitidas de geração em geração (práticas culturais), no âmbito das quais se modificam, constroem e se estabelecem as funções reforçadora e aversiva de vários eventos (SKINNER, 2007). Por fim, é no nível ontogenético que é estabelecido o repertório comportamental do indivíduo, diferenciando-o de outros organismos da mesma espécie e de outros indivíduos que também fazem parte da cultura. Esse repertório comportamental é constituído tanto por reflexos condicionados quanto por comportamentos operantes, resultantes de processos de aprendizagem como o condicionamento reflexo e o condicionamento operante, respectivamente.

Para a Análise do Comportamento, todo o comportamento individual, seja ele considerado "normal" ou "anormal" é explicado pelos mesmos processos de aprendizagem. Pensando nisso, faz-se necessário compreender qual a visão analítico-comportamental de psicopatologia. Banaco, Zamignani e Meyer (2010) apresentam uma diferenciação de três modelos científicos utilizados para explicar a psicopatologia: o modelo médico, o modelo quasemédico e o modelo analítico-comportamental ou psicológico.

O modelo médico pauta seu tratamento na utilização de fármacos (BANACO; ZAMIGNANI; MEYER, 2010), tratando o transtorno como resultado de uma patologia biológica subjacente (GONGORA, 2003). Já o modelo quasemédico é adotado por teorias psicológicas que defendem a existência de uma dicotomia entre o normal e o patológico, divergindo apenas na forma de tratamento, uma vez que nem sempre adotam a utilização de fármacos (BANACO; ZAMIGNANI; MEYER, 2010). Esses dois modelos compartilhariam ainda de uma visão internalista da psicopatologia, sendo considerada como uma doença biológica que estaria pré-determinada ao indivíduo (PRADO, 2013). Para diagnosticar essas doenças são utilizados manuais classificatórios de transtornos mentais, como a Classificação Internacional de Doenças (CID) da Organização Mundial de Saúde (1997), e o Manual de Diagnóstico e Estatística das Perturbações Mentais (DSM) da Associação Americana de Psiquiatria (2014) (PRADO, 2013).

O modelo analítico-comportamental, por seu turno, entende que não existe um comportamento que seja considerado patológico em si mesmo, pois, a rigor, a emissão de qualquer comportamento é explicada voltando-se para o contexto atual e histórico, atentando-se, sobretudo, para as suas consequências (no caso do comportamento operante), e para os estímulos eliciadores incondicionados e condicionados (no caso do comportamento respondente) (GONGORA, 2003). Sendo assim, os critérios que determinariam 
se o comportamento é patológico não residem em um modo de funcionamento do comportamento considerado anormal, mas nas normas culturais de uma dada sociedade, que classificam (e valoram) o comportamento do indivíduo (e o próprio indivíduo) como anormal, dispondo contingências reforçadoras e punitivas em função dessa classificação (GONGORA, 2003).

Dessa perspectiva, o conceito de anormalidade tem a função social de distinguir como "desvio" tudo que for contrário a padrões previamente estabelecidos em uma dada cultura. Essa distinção é sancionada por meio de rótulos diagnósticos, que acabam por determinar a forma com que as pessoas devem agir e quem elas são (ULMMANN; KRASNER, 1969). Os rótulos possuem como principal meio de propagação os manuais classificatórios anteriormente mencionados, como o CID e o DSM.

A Análise do Comportamento critica a utilização desses manuais de forma isolada, uma vez que os diagnósticos são pautados na descrição da frequência e topografia do "comportamento-problema" sem que seja analisada a função desse comportamento (BANACO; ZAMIGNANI; MEYER, 2010). O fato de a Análise do Comportamento não subscrever uma concepção internalista e essencialista de psicopatologia não implica na desconsideração de que podem emergir comportamentos que provocam sofrimento para o indivíduo e/ou para aqueles de seu entorno, de modo que esse indivíduo poderá requerer auxílio psicológico. A diferença é que esse comportamento será entendido e explicado por meio de uma análise funcional, que busca descrever e alterar as variáveis das quais esse comportamento é função. Tais variáveis encontram-se no contexto imediato e histórico; lembrando que esses contextos são complexos, uma vez que podem envolver aspectos filogenéticos, ontogenéticos e culturais. $\mathrm{O}(\mathrm{a})$ analista do comportamento poderá até se valer de manuais diagnósticos como ponto de partida, mas não de chegada, para realizar a análise funcional, compartilhando também, por meio do uso desses manuais, de termos comuns com outros profissionais da saúde, desde que, novamente, esse diálogo seja perpassado por uma perspectiva contextualista daquilo que está sendo tratado como "comportamento-problema" (BANACO; ZAMIGNANI; MEYER, 2010).

Considerando a alta incidência de diagnósticos de ansiedade no Brasil, com aproximadamente 20 milhões de brasileiros diagnosticados, e que 0 comportamento ansioso pode acarretar uma série de dificuldades ao indivíduo, em diferentes esferas da vida (profissional, familiar, afetiva, social, etc.), como a Análise do Comportamento tem examinado o assunto? $\mathrm{O}$ objetivo, então, deste trabalho é apresentar um panorama a respeito de como a literatura analítico-comportamental nacional tem discutido a ansiedade.

\section{METODOLOGIA}

Foi produzida uma pesquisa bibliográfico-exploratória cujas fontes foram artigos científicos publicados em periódicos nacionais especializados em Análise do Comportamento, sendo eles: Revista Brasileira de Terapia Comportamental e Cognitiva (RBTCC), Revista Brasileira de Análise do Comportamento (REBAC) e Perspectivas em Análise do Comportamento (PAC). 
Para isso, foi realizado o download de todos os volumes e números dos periódicos mencionados anteriormente, começando pelo volume mais antigo disponibilizado online, de 1999, da RBTCC, indo até o último volume publicado, pelos periódicos, à época da busca bibliográfica, que foi realizada em março de 2019, perfazendo um período de vinte anos. Um conjunto de termos que se referiam à ansiedade, e aos subtipos de transtornos de ansiedade existentes de acordo com o DSM-V (2014), foram delimitados para serem utilizados na busca e seleção das fontes: ansiedade, transtorno de ansiedade, transtorno de ansiedade de separação, mutismo seletivo, fobia específica, transtorno de ansiedade social, transtorno de pânico, agorafobia, transtorno de ansiedade generalizada, transtorno de ansiedade induzido por substância/medicamento, transtorno de ansiedade devido a outra condição médica, transtorno de ansiedade especificado e transtorno de ansiedade não especificado.

Por meio do mecanismo $C R T L+F$, esses termos foram buscados no título, resumo, palavras-chave e/ou no corpo do texto. Os textos que apresentavam ao menos uma das palavras relativas à ansiedade no título, resumo e/ou nas palavras-chaves eram selecionados para leitura preliminar, e submetidos a ulterior averiguação de sua pertinência como fonte da pesquisa, conforme critérios de inclusão e exclusão que serão descritos adiante. Os textos selecionados foram catalogados em uma tabela que requisitava as seguintes informações: (i) a citação em que continha as palavras relacionadas à terminologia da ansiedade; (ii) o contexto/conceito apresentado na citação; (iii) qual a contribuição para a compreensão da ansiedade como fenômeno comportamental; e, por fim, (iv) qual o nível de análise de discussão da ansiedade (filogenético, ontogenético ou cultural).

A análise do material foi separada em duas etapas. A primeira delas consistiu em uma descrição quantitativa, representada por um fluxograma que sistematizou a quantidade de artigos encontrados por periódicos, quantos foram excluídos e a justificativa da exclusão. A segunda compreendeu uma análise interpretativa, definida pela elaboração de categorias de análise que tinham como objetivo sistematizar as principais informações tratadas pela literatura analítico-comportamental sobre a ansiedade.

O material foi selecionado de acordo com os seguintes critérios de inclusão: ocorrência dos termos relacionados à ansiedade, e aos transtornos associados a ela, no título, resumo, palavras-chave e/ou no corpo do texto, e artigos redigidos em português. Já os critérios de exclusão foram definidos por materiais bibliográficos que não se baseavam na teoria comportamentalista radical para explicar a ansiedade (e.g., teoria cognitivo-comportamental); artigos redigidos em outro idioma, que não o português; materiais que não eram artigos como, por exemplo, editoriais e resenhas de livro; textos que não discutiam a ansiedade, ou aqueles que apenas citavam os termos sem realizar uma discussão mais pormenorizada.

\section{RESULTADOS E DISCUSSÃO}

O processo de busca e seleção das fontes está representado na Figura 1. Foi realizado o download de todas as edições dos periódicos especializados em Análise do Comportamento selecionados, que, por sua vez, foram salvos 
em pastas previamente nomeadas com o título da revista, o volume e sua edição. Posteriormente, por meio do mecanismo $C R T L+F$, foram selecionadas para leitura publicações que continham no título, no resumo e/ou nas palavraschave termos que se relacionassem à ansiedade e seus subtipos determinados pelo DSM-V (2014).

Diante disso, o material escolhido foi direcionado para outras pastas específicas nomeadas com as respectivas palavras identificadas nos artigos. Entre todos os periódicos, foram encontrados 157 artigos que mencionavam as palavras ansiedade e/ou pânico e/ou agorafobia. Do total de 157 artigos, 119 estavam presentes no periódico RBTCC, revista que também aceita artigos que são orientados pela teoria cognitivo-comportamental. Em vista disso, 33 artigos foram excluídos por não serem da abordagem analítico-comportamental, e 76 artigos foram excluídos por apresentarem uma das três palavras apenas uma vez no corpo do texto, não apresentando uma maior discussão sobre o tema. Por esse motivo, esses artigos foram considerados com quantidade insuficiente de termos. Sendo assim, foram selecionados para leitura 10 artigos da RBTCC.

No periódico REBAC foram encontrados 12 artigos que continham uma das palavras previamente mencionadas. Desse total, 11 foram eliminados também por insuficiência de ocorrência de termos, restando apenas 1 artigo para leitura neste periódico.

Por fim, no periódico PAC foram encontrados 26 artigos que continham uma das palavras mencionadas, porém 22 textos foram eliminados pelo critério de insuficiência de aparição dos termos, restando, então, 4 artigos da PAC para leitura. Assim sendo, 15 artigos foram selecionados para leitura.

Para realização da análise interpretativa os 15 artigos foram lidos e documentados. Com base na compilação do material selecionado, conforme 0 modelo de tabela adotado, foram construídas categorias de análise que buscaram dar relevo aos principais temas discutidos pelos textos selecionados quando o assunto em pauta era a ansiedade. As categorias de análise, que serão descritas, a seguir, são: concepções tradicionais de ansiedade criticadas pela Análise do Comportamento; definição de ansiedade para teoria analíticocomportamental; outras variáveis associadas à ansiedade; ansiedade normal e patológica; formas de tratamento da ansiedade na Análise do Comportamento; e, por fim, o lugar da cultura nos estudos analíticos-comportamentais nacionais sobre ansiedade.

A primeira categoria descreve críticas da Análise do Comportamento a determinadas concepções de ansiedade, o que dá ensejo para entender, por meio da segunda categoria, como a teoria analítico-comportamental define e explica da ansiedade. A terceira categoria descreve outras variáveis invocadas pelos estudos analítico-comportamentais para discutir a ansiedade, como gênero e necessidades especiais. Além disso, foram identificados, na literatura selecionada, dois tipos de ansiedade, a normal e a patológica, em que a última é vista como motivo de queixa clínica; distinções essas que serão pormenorizadas na descrição da quarta categoria. Já a quinta categoria arrola alguns modelos e técnicas psicoterápicas utilizadas pela Análise do Comportamento para o tratamento de ansiedade, que foram citadas nos textos examinados. A última categoria mostra que a ansiedade, foi, então, predominantemente analisada no contexto ontogenético, porém, argumenta-se 
sobre a necessidade de direcionar os estudos sobre o tema para o nível cultural.

Figura 1 - Representação do processo de busca e seleção de artigos sobre ansiedade publicados nos periódicos especializados em Análise do Comportamento ${ }^{1}$ :

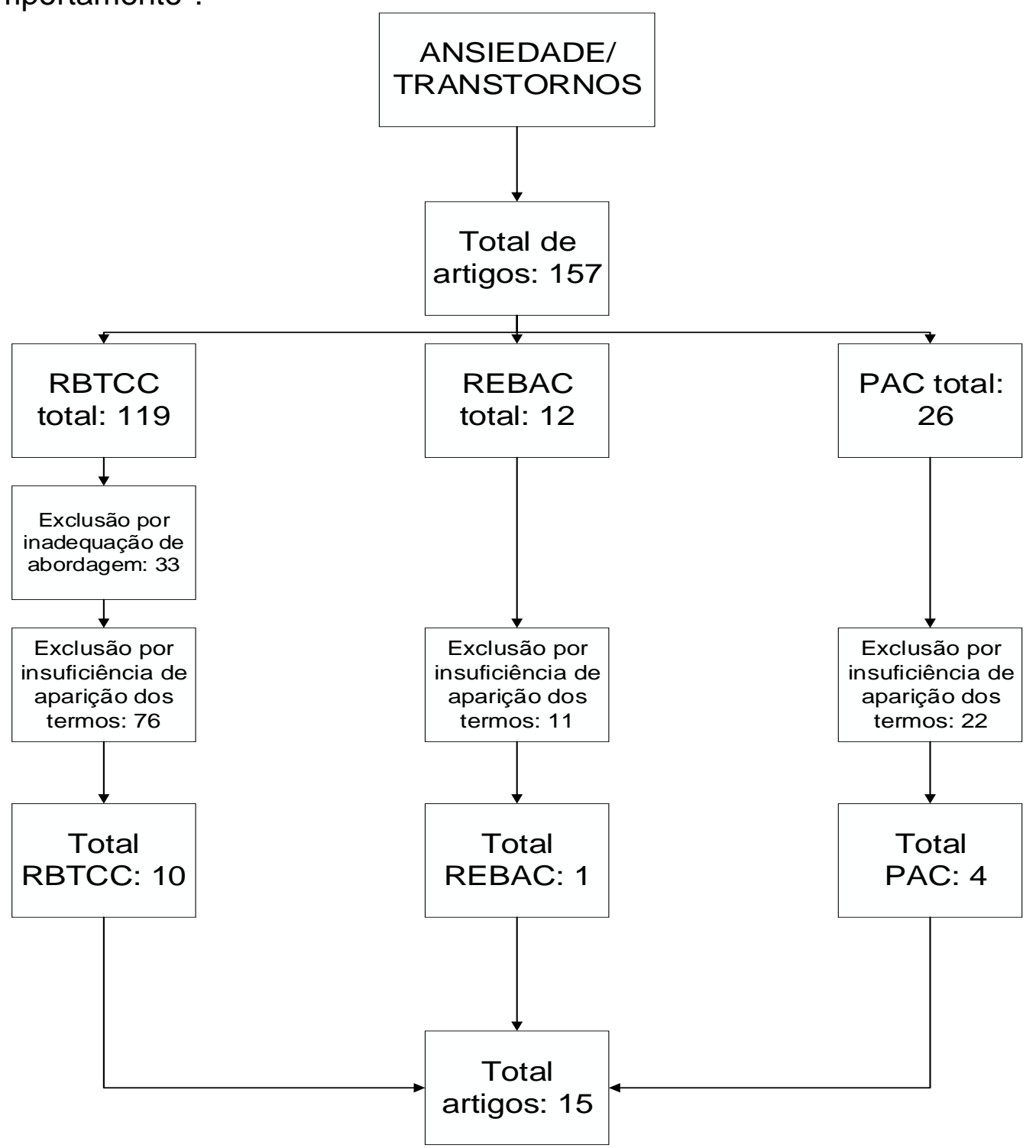

Notas: ${ }^{1}$ Revista Brasileira de Terapia Comportamental e Cognitiva (RBTCC), Revista Brasileira de Análise do Comportamento (REBAC), e Perspectivas em Análise do Comportamento (PAC).

Fonte: os autores

\section{Concepções tradicionais de ansiedade criticadas pela Análise do} Comportamento

Dentre os artigos selecionados, 5 deles mencionaram definições e/ou explicações da ansiedade que são alvo de críticas da Análise do Comportamento. A ansiedade é comumente entendida como uma perturbação provocada devido a um evento futuro, ou seja, é explicada por meio de uma explicação teleológica que define esse fenômeno como uma "expectativa" de 
que algo irá acontecer (REGIS NETO et al., 2011). A Análise do Comportamento não compactua com essa definição tradicional de ansiedade, por entender que um evento que ainda não ocorreu não seria capaz de alterar a probabilidade de ocorrência de um dado tipo de resposta no presente. O que explica essa alteração está no passado e não no futuro, mais especificamente, em uma história prévia de reforçamento, que aumenta a probabilidade de uma ação semelhante voltar a acontecer no futuro (REGIS NETO et al., 2011).

Explicações internalistas da ansiedade foram outro alvo de crítica da Análise do Comportamento. Essa forma de análise decorre da frequente afirmação de que o comportamento ansioso (e o comportamento de esquiva que o caracteriza) ocorre devido a fontes internas de controle (SIDMAN, 2009). De acordo com Sidman (2009), isso acontece, pois, ao experienciar estados internos como os sentimentos e emoções, os indivíduos tendem a atribuir a eles um status causal, negligenciando as relações entre essas condições corporais e os eventos aversivos presentes no ambiente. Os sujeitos nem sempre conseguem identificar e descrever verbalmente esses eventos aversivos, o que não impede que emitam comportamentos de esquiva, e acabem incorrendo em explicações internalistas do comportamento ansioso (SIDMAN, 2009).

Além disso, outros 2 artigos criticaram a utilização de manuais de diagnóstico, como o DSM-V, para classificação de diferentes transtornos, uma vez que esses manuais empregam uma análise topográfica, e não funcional (SOUSA, 2003; ZAMIGNANI; BANACO, 2005). Ao realizar uma análise topográfica, apenas o comportamento ansioso estaria sendo observado, ignorando as variáveis responsáveis por manter tal comportamento. A despeito disso, foi pontuado que os manuais podem ser usados com ressalvas, como um facilitador de comunicação entre psicólogos(as) e profissionais de outras áreas da saúde (SOUSA, 2003).

\section{Definição de ansiedade para a Análise do Comportamento}

Dos 15 artigos selecionados, 7 apresentaram uma definição da ansiedade para a Análise do Comportamento. Dentre esses 7 artigos, 2 a mencionaram como um fenômeno complexo (ZAMIGNANI; BANACO, 2005; FERREIRA et al., 2010); 4 utilizaram de modelos experimentais para explicar a ansiedade na perspectiva analítico-comportamental (NEVES et al., 1999; ZAMIGNANI; BANACO, 2005; REGIS NETO et al., 2011; SANCHEZ; GOUVEIA-JUNIOR, 2011); outros 2 artigos destacaram a importância dos ambientes sociais na compreensão da manutenção dos comportamentos ansiosos (MORAES et al., 2006; OLIVEIRA; DUARTE, 2004); e, por fim, dos 7 artigos, 3 mencionaram a cultura (ABREU; PRADA, 2004; HENKLAIN; CARMO, 2011; SOUSA, 2003).

A Análise do Comportamento entende a ansiedade como um fenômeno comportamental complexo (FERREIRA et al., 2010). Essa visão ocorre, pois, ao compreender o comportamento como um produto de contingências filogenéticas, ontogenéticas e culturais, reconhece-se que relações comportamentais podem incluir variáveis de um ou mais níveis, determinando assim o grau de complexidade do fenômeno, estabelecendo-se um continuum de complexidade (FERREIRA et al., 2010). Sendo assim, a complexidade do 
fenômeno será menor se envolver apenas um dos níveis, aumentando seu grau de complexidade de acordo com a abrangência de outros níveis, ou seja, o fenômeno que envolver variáveis relacionadas aos três níveis será o mais complexo (FERREIRA et al., 2010).

De um ponto de vista filogenético, a ansiedade pode ser compreendida a partir de dois tópicos: as condições fisiológicas entendidas como subprodutos de contingências específicas e a possibilidade dessa condição fisiológica adquirir uma função comportamental (FERREIRA et al., 2010). No que diz respeito às condições fisiológicas, os autores afirmam que os responsáveis por produzir determinada condição fisiológica na ansiedade são os eventos aversivos e pré-aversivos (FERREIRA et al., 2010). Em relação ao segundo tópico, os autores argumentam que os componentes orgânicos podem se inserir nas relações comportamentais, adquirindo alguma função, como, por exemplo, um estímulo discriminativo de respostas verbais ou não verbais (FERREIRA et al., 2010).

No nível ontogenético, a ansiedade ou o comportamento ansioso é compreendido em termos de componentes respondentes e operantes. Os componentes respondentes do comportamento ansioso são explicados como respostas reflexas condicionadas produzidas pelo processo de condicionamento respondente ou pavloviano.

No condicionamento respondente, o estímulo eliciador incondicional de uma resposta é antecedido pela apresentação de um estímulo considerado neutro (REGIS NETO et al., 2011). A partir disso, o estímulo previamente considerado neutro irá adquirir propriedades eliciadoras similares ao do estímulo incondicional, passando a eliciar, então, uma resposta condicionada (REGIS NETO et al., 2011). O condicionamento respondente auxilia na adaptação do organismo, preparando-o para o estímulo eliciador incondicional, pois, "essa relação de 'antecipação' ocorre porque na história desse organismo o estímulo presente [...] antecipou sistematicamente o estímulo incondicional [...], tornando-se um eliciador condicional." (REGIS NETO et al., 2011, p. 7).

Ao entender que a antecipação é um componente do estímulo aversivo que compõe a definição de ansiedade, faz-se necessário compreender o conceito de estímulo aversivo condicionado. Para Zamignani e Banaco (2005)

[...] estímulos habitualmente neutros para a resposta de medo, se forem seguidos consistentemente por (ou seja, se forem condicionados a) estímulos aversivos incondicionados, passarão a adquirir, eles próprios, propriedades aversivas e poderão eliciar respostas "semelhantes ao medo": a ansiedade [...]. Esta, então, seria uma resposta que se "anteciparia" ao estímulo aversivo incondicionado, embora não estivesse sob controle do futuro, e sim de um estímulo aversivo condicionado presente (chamado por alguns autores como 'pré-aversivos'). (p. 82)

A resposta de ansiedade é, portanto, controlada por um evento aversivo condicionado e não por um evento futuro. Mas uma explicação analíticocomportamental da ansiedade não se limita à descrição de sua dimensão reflexa. O comportamento ansioso também é composto por respostas que modificam o ambiente em que estão presentes, ou seja, por respostas operantes. Alguns modelos experimentais de ansiedade, citados pela literatura 
consultada, ajudam a compreender a interação entre a dimensão reflexa e a dimensão operante do comportamento ansioso, como é o caso da supressão condicionada. Esse modelo mostra que "caso não haja a possibilidade de respostas de fuga e esquiva, o efeito reflexo da estimulação condicionada paralisa a emissão de respostas operantes que produzem o estímulo reforçador positivo." (ZAMIGNANI; BANACO, 2005, p. 83). Assim, na presença de determinados estímulos aversivos condicionados, pode ocorrer uma supressão temporária da emissão de respostas operantes (REGIS NETO et al., 2011). Em situações eliciadoras do comportamento ansioso, determinados comportamentos operantes são suprimidos devido à ocorrência de respostas biológicas incompatíveis, desencadeadas por eventos aversivos, como a sudorese e a taquicardia.

Além disso, para compreender a ansiedade, é preciso entender o conceito de eventos privados, ou seja, estímulos que não são passíveis de observação direta a outras pessoas, sendo acessíveis apenas ao indivíduo (FERREIRA et al., 2010). Para Skinner (2006), existe uma parte dos eventos que ocorrem dentro da pele de cada indivíduo (uma condição corporal) que pode adquirir função de estímulo (FERREIRA et al., 2010). Mas, vale destacar, que "nem todo evento corporal é um estímulo (seja ele público ou privado) [...]." (FERREIRA et al., 2010, p. 72). Essa condição corporal pode adquirir uma função de estímulo dadas certas contingências: "[...] o sujeito é exposto a uma contingência que é responsável por eliciar o estado corporal, e pode relatar qual é esse estado diante de uma audiência, que controla as especificidades do relato." (CUNHA; BORLOTI, 2009, p. 213).

Alguns eventos corporais frequentemente descritos como negativos (taquicardia, hiperventilação, sudorese, dores, tremores e sensação de sufocamento) são denominados como ansiedade, porém não podem ser considerados a única variável (ZAMIGNANI; BANACO, 2005). É preciso compreender que é a partir de uma comunidade verbal que o indivíduo pode responder discriminativamente sob controle de determinadas condições corporais (FERREIRA et al., 2010). Embora a descrição de sentimentos seja relevante, o mais importante é "enfatizar que respostas verbais autodescritivas, como 'estou ansioso', podem ficar sob controle de estímulos privados equivalentes ou correlacionados com estímulos públicos." (FERREIRA et al., 2010, p. 73). Além disso, "é possível que estimulações fisiológicas eliciem as respostas de medo: a pessoa responde negativamente aos seus estados corporais." (BRITTO; DUARTE, 2004, p. 166).

Alguns comportamentos operantes controlados por reforçamento negativo, como a fuga e a esquiva, são considerados característicos da ansiedade (ZAMIGNANI; BANACO, 2005). A esquiva é uma forma de postergar ou eliminar o contato com um estímulo aversivo condicionado, enquanto a fuga é uma resposta que elimina ou diminui a intensidade de um estímulo incondicional aversivo (SKINNER, 2003). O comportamento ansioso pode, ainda, ocorrer por "[...] reforçamento positivo se, por exemplo, a resposta de esquiva for seguida por um reforçador social." (ZAMIGNANI; BANACO, 2005, p. 84).

O conceito de operação estabelecedora descreve que determinadas condições podem gerar uma mudança na efetividade reforçadora de outros 
eventos, como, por exemplo, a privação (FERREIRA et al., 2010). Indivíduos que se encontram privados de reforçadores sociais, como a atenção, ao emitirem comportamentos ansiosos podem receber atenção, gerando reforçadores positivos, mantendo-se, assim, comportamentos compatíveis com a ansiedade, em função do reforçamento positivo social.

Os ambientes sociais apareceram como fonte de controle do comportamento ansioso em dois artigos (MORAES et al., 2006). No contexto de exposições orais em universitários, a maneira com que os professores se comportavam diante dos alunos modificavam os comportamentos emitidos pelos estudantes. O nível de ansiedade dos alunos foi medido utilizando a Escala de Medo de Avaliação Negativa, o Inventário de Ansiedade (IDATE) e a medida da Frequência Cardíaca, demonstrando ser maior na presença de professores ansiogênicos, que emitiam comportamentos punitivos, do que os não ansiogênicos (OLIVEIRA; DUARTE, 2004). A presença de professores punitivos pode, então, funcionar como ambientes sociais aversivos para 0 comportamento de alunos, afetando a probabilidade de ocorrência de respostas de ansiedade na presença deles.

Além do ambiente escolar, outros ambientes sociais foram explorados pela literatura consultada, como na situação de tratamentos odontológicos, demonstrando que mães que emitiam comportamentos ansiosos podiam influenciar no comportamento ansioso de seus filhos (MORAES et al., 2006), uma vez que filhos de mães com alto nível de ansiedade emitiam mais comportamentos ansiosos quando comparado a filhos de mães pouco ansiosas (MORAES et al., 2006). Essas afirmações permitem questionamentos como, se os ambientes sociais podem afetar a probabilidade de emissão de comportamentos ansiosos, as práticas culturais seriam fatores a serem considerados no entendimento de respostas de ansiedade dos indivíduos.

O artigo "Produção analítico-comportamental sobre ensino aprendizagem de habilidades matemáticas: Dados representativos de eventos científicos brasileiros" mencionou a relação entre a cultura e o ensino de habilidades matemáticas, apresentando uma revisão de estudos com informações de 19 anos de publicações sobre o tema. A matemática é uma matéria considerada difícil e, ao mesmo tempo, é socialmente vista como essencial para um bom desempenho acadêmico, o que pode ocasionar respostas ansiosas nos estudantes (HENKLAIN; CARMO, 2011). As autoras sugerem uma influência da cultura no comportamento dos indivíduos em relação ao ensino e aprendizagem da matemática, que são perpassados por exigências do mundo moderno que podem funcionar como estimulação aversiva, gerando, em determinadas ocasiões, comportamentos ansiosos (HENKLAIN; CARMO, 2011).

Ao entrar em um contexto universitário, os(as) alunos(as) são frequentemente requisitados para exibir determinação, liderança e competência. Oliveira e Duarte (2004) afirmam que ao se deparar com todas as imposições sociais para que se consiga um emprego, sabendo que nem todos terão a mesma oportunidade, surge uma maior exigência, gerando desconforto e, até mesmo, ansiedade. Sendo assim, os autores argumentam que "a ansiedade excessiva frente a situações de interação social e exposição está relacionada a uma forte motivação para causar boa impressão nos outros, bem 
como a dúvidas acerca da própria habilidade em conseguir tal impressão." (OLIVEIRA; DUARTE, 2004, p. 185). Nesse contexto, comportamentos punitivos, tais como vaias e comentários negativos por parte dos espectadores, podem ter função aversiva para os(as) alunos(as), podendo ocasionar respostas de ansiedade em situações de interação social.

\section{Outras variáveis associadas à ansiedade}

A ansiedade apareceu associada a outras variáveis, como em casos de pessoas com algum tipo de limitação auditiva. Esse tema apareceu em 2 dos 15 artigos selecionados (SANCHEZ; GOUVEIA-JUNIOR, 2008, 2011).

Um dos instrumentos utilizados para mensurar a ansiedade é a Escala Analógica de Humor (EAH). Porém, Sanchez e Gouveia-Junior (2008) destacaram a necessidade de adaptá-lo para pessoas surdas. A utilização de instrumentos como forma de auxílio nas avaliações psicológicas é fundamental para que possa fazer uma análise detalhada.

Além do $E A H$, outros instrumentos são utilizados, como o Inventário de Ansiedade Traço-Estado (IDATE) e o teste de simulação do falar em público (SANCHEZ; GOUVEIA-JUNIOR, 2011). O IDATE exige uma maior compreensão da língua portuguesa, língua esta que os indivíduos com deficiência auditiva apresentam uma maior dificuldade de compreensão e escrita, o que impede um resultado fidedigno do teste (SANCHEZ; GOUVEIAJUNIOR, 2011). O teste de simulação do falar em público, por sua vez, demonstrou um aumento do nível de ansiedade em indivíduos que não apresentavam traços ansiosos, pois induz a ansiedade independente do traço de ansiedade, ou seja, o resultado não deve variar de acordo com a língua utilizada, podendo ser utilizado em LIBRAS (SANCHEZ; GOUVEIA-JUNIOR, 2011).

Outras variáveis parecem estar relacionadas ao diagnóstico de ansiedade, como a diferença de pessoas acometidas com o transtorno de acordo com seu gênero (SANCHEZ; GOUVEIA-JUNIOR, 2011), visto que o público feminino apresenta uma taxa de transtornos quase duas vezes maior que o masculino (WHO, 2017). Sanchez e Gouveia-Junior (2011) apontaram também que a ansiedade pode estar relacionada com a idade dos indivíduos, indicando que embora possa ocorrer em diferentes períodos do desenvolvimento, ela aparenta estar mais acentuada nos adolescentes, por ser uma fase que é perpassada por série de mudanças físicas, biológicas e psicológicas.

\section{Ansiedade normal e patológica}

Entre os 15 artigos analisados, 3 mencionaram dois tipos de ansiedade, a normal e a patológica. A ansiedade pode estar relacionada a diferentes eventos, como estados internos de quem relata e os processos comportamentais responsáveis por desencadear esses estados internos (ZAMIGNANI; BANACO, 2005). Desse modo, eventos descritos como agradáveis podem eliciar um sentimento de ansiedade em momentos que exigem algum tipo de espera (ZAMIGNANI; BANACO, 2005). Esse tipo de ansiedade é denominado ansiedade normal (OLIVEIRA; DUARTE, 2004), pois 
"a ansiedade normal é um estado emocional presente em todos os seres humanos [...]." (SANCHEZ; GOUVEIA-JUNIOR, 2011, p. 23).

A ansiedade é considerada patológica e analisada como fenômeno clínico quando impedir a realização de atividades cotidianas do indivíduo, quando envolver um grau de sofrimento considerado significativo, e quando a emissão de respostas de fuga e esquiva forem frequentes (ZAMIGNANI; BANACO, 2005). Em outros dois artigos (ABREU; PRADA, 2004; SOUSA, 2003), a ansiedade foi também associada a outros transtornos psicológicos, como no caso do Transtorno de Ansiedade Obsessivo-Compulsivo (TOC) e o Transtorno de Personalidade Borderline. No caso de TOC, foi encontrado um alto nível de ansiedade diante das obsessões típicas do transtorno, como os pensamentos e ideias, apontando ainda para um elevado sofrimento (ABREU; PRADA, 2004). É possível analisar, então, que nos pacientes com TOC, as respostas compulsivas são comportamentos que possuem função de esquiva de situações eliciadoras de ansiedade (ABREU; PRADA, 2004). A agorafobia, um subtipo de ansiedade listado no DSM-V (2014), foi mencionada em um artigo sobre o Transtorno de Personalidade Borderline (SOUSA, 2003), no qual o paciente diagnosticado emitia, também, comportamentos compatíveis com esse subtipo de ansiedade.

Considerando a noção de ansiedade patológica, é preciso identificar possíveis formas de tratamento para o comportamento ansioso.

\section{Formas de tratamento da ansiedade na Análise do Comportamento}

Como forma de tratamento para os transtornos de ansiedade, Pezzato, Brandão e Oshiro (2012) defenderam o uso de procedimentos que tornem o processo terapêutico menos aversivo e mais reforçador, apontando para a necessidade de uma relação reforçadora entre 0 cliente e o terapeuta (ZAMIGNANI; BANACO, 2005).

Além disso, a Psicoterapia Analítica Funcional (FAP) pode ser utilizada como uma forma de tratamento por se caracterizar não apenas como instrumento de intervenção, mas como uma estratégia que possibilitaria maior adesão do cliente à terapia (PEZZATO; BRANDÃO; OSHIRO, 2012), sendo também eficiente em indivíduos que não se adequam aos modelos de terapia tradicionais, como os que se esquivam de relacionamentos pessoais (PEZZATO; BRANDÃO; OSHIRO, 2012), que é o caso de pacientes ansiosos.

De acordo com o que foi visto anteriormente, os estados corporais negativos podem ser eliciadores de respostas ansiosas, como, por exemplo, a hiperventilação, que consiste em uma respiração rápida e ineficiente, sendo um dos sintomas mais frequentes em indivíduos ansiosos. Diante disso, um artigo mencionou uma proposta de intervenção de treinamentos de respiração diafragmática, bem como técnicas de relaxamento muscular (OLIVEIRA; DUARTE, 2004).

Foi destacada também a necessidade de analisar o comportamento não apenas do cliente, mas também do terapeuta, identificando-se inter-relações entre eles, bem como seus respectivos comportamentos verbais (NOBILE; GARCIA; BOLSONI-SILVA, 2017). Para isso, os autores adotaram o uso do sistema de interação terapêutica elaborado por Zamignani, denominado Sistema Multidimensional de Categorização de Comportamento da Interação 
Terapêutica (SiMCCIT) como forma de categorizar o comportamento de ambos (NOBILE; GARCIA; BOLSONI-SILVA, 2017).

Como já foi mencionado, a ansiedade pode ser considerada um fenômeno complexo, podendo envolver variáveis de diferentes níveis de variação e seleção pelas consequências, dispostas em um continuum de complexidade (FERREIRA et al., 2010). Dessa perspectiva, as intervenções deveriam focar nas diferentes relações envolvidas no comportamento, ou seja, a escolha da intervenção mais apropriada decorreria de uma análise funcional de cada um dos três níveis envolvidos nesse continuum e quais as possibilidades de relação entre eles (FERREIRA et al., 2010).

Analisando a ansiedade como um fenômeno comportamental complexo, que pode envolver os três níveis de análise (filogenético, ontogenético e cultural), é preciso questionar como a literatura analítico-comportamental nacional abordou a cultura na explicação da ansiedade.

\section{O lugar da cultura nos estudos analíticos-comportamentais nacionais sobre ansiedade}

Dentre os 15 artigos analisados, apenas 3 mencionaram a cultura de alguma forma. Uma das menções à cultura estava relacionada à noção de ansiedade como um fenômeno complexo, que poderia envolver análise os três níveis de seleção, a filogênese, a ontogênese e a cultura (FERREIRA et al., 2010), sendo considerada mais complexa se abarcasse todos esses níveis.

A cultura também foi invocada no âmbito da discussão sobre eventos privados que podem participar do comportamento ansioso. Condições corporais envolvidas na ansiedade podem adquirir função em contingências sociais verbais que ensinam o indivíduo a relatar seu comportamento sob controle dessas condições. Essas contingências sociais verbais fazem parte de práticas culturais ocidentais, que ensinam o indivíduo a descrever o estímulo que está "[...] localizado no próprio corpo do indivíduo." (FERREIRA et al., 2010, p. 71). A partir disso, a Análise do Comportamento se interessa pela cultura pois,

[...] na medida em que a cultura ocidental, particularmente, estimula os indivíduos a se comportarem de forma "introspectiva", ensinamos a relatar sentimentos como causas de seus comportamentos e a comportar-se discriminativamente sob controle dessas falas. (FERREIRA et al., 2010, p. 71)

Além de a dimensão cultural ter sido mencionada na explicação do ensino de autorrelatos sob controle de condições corporais, envolvidas na ansiedade, a cultura também apareceu relacionada à ansiedade na forma de práticas culturais competitivas que podem ser fonte de comportamentos ansiosos. Pautando-se em Zimbardo, Oliveira e Duarte (2004) argumentaram que "[...] a competição e a realização individual excessivamente valorizadas nos dias atuais, podem dar origem à ansiedade social." (p. 184).

O nível cultural foi, então, mencionado em $20 \%$ dos artigos analisados, o que sugere que a cultura não está sendo analisada de forma tão abrangente na literatura analítico-comportamental nacional. 
De acordo com Skinner (2003), todo comportamento humano é, também, produto de uma história cultural. A cultura definida, então, como "[...] interações comportamentais entre indivíduos (contingências sociais), torna-se, ela mesma, entrelaçamento de contingências de reforçamento (de comportamentos) e seus produtos, portanto, nosso legítimo objeto de estudo." (ANDERY, 2011, p. 207). Dessa forma, tanto a cultura quanto as práticas culturais são fenômenos comportamentais, pois, comportamentos individuais constituem tais práticas, sendo assim, a cultura é um fenômeno supracomportamental que precisa ser compreendido para que então possa compreender o próprio comportamento humano (ANDERY, 2011). Pensando nisso, surge a Análise Comportamental da Cultura, uma área da Análise do Comportamento que se propõe a estudar os fenômenos sociais ou a cultura como foco de seus trabalhos, colocando-a no papel de variável dependente, e não mais independente (ANDERY, 2011).

Tendo em vista que o Brasil foi considerado o país mais ansioso do mundo pela WHO em 2017 e, novamente, em 2019 por essa mesma organização, o transtorno de ansiedade aparenta ter tomado uma proporção cultural, e não só individual, visto que aproximadamente 20 milhões de brasileiros sofrem de ansiedade, o que sugere a necessidade de uma maior compreensão do papel da cultura no comportamento ansioso.

\section{CONSIDERAÇÕES FINAIS}

Este artigo buscou sistematizar como a literatura analíticocomportamental nacional tem discutido a ansiedade. Com base no exame dos 15 artigos selecionados, foi possível identificar críticas proferidas pela Análise do Comportamento a concepções teleológicas e internalistas de ansiedade, além de ter indicado os limites do uso exclusivo de manuais diagnósticos para compreender e tratar a ansiedade. Além disso, a literatura analíticocomportamental tem definido a ansiedade como um fenômeno comportamental complexo, em que variáveis mantenedoras do comportamento ansioso podem abarcar os três níveis de variação e seleção (filogenético, ontogenético e cultural). Não obstante a consideração dessa complexidade, a literatura consultada concentrou sua discussão no nível ontogenético.

Nesse âmbito, o comportamento ansioso envolve componentes respondentes e operantes que podem interagir entre si. De um modo geral, a função do comportamento ansioso é esclarecida pela evitação do contato com estímulos aversivos condicionados, por meio do comportamento de esquiva. Mas, além do reforçamento negativo, o comportamento ansioso pode também ser mantido por reforçamento positivo. Além disso, operações estabelecedoras podem afetar a probabilidade de emissão de comportamentos ansiosos, alterando 0 valor reforçador de alguns eventos que mantêm esses comportamentos, como os reforçadores sociais. Outras variáveis controladoras do comportamento ansioso são os eventos privados, na forma de condições corporais, que assumiram função de estímulos em determinadas contingências sociais verbais, e que passam a controlar relatos verbais sobre se sentir ou estar ansioso. 
A ansiedade pode ser considerada normal ou motivo de queixa clínica, a depender do nível de comprometimento que acarreta para a vida do indivíduo. Nesse último caso, a Análise do Comportamento também dispõe de modelos e técnicas psicoterápicas para lidar com o comportamento ansioso.

É importante destacar que este artigo apresenta suas limitações, visto que o material analisado se restringiu a artigos publicados em veículos nacionais especializados em Análise do Comportamento, não abrangendo revistas não especializadas, livros e capítulos de livro, teses de mestrado e/ou doutorado e a literatura internacional. Porém, analisando os dados e as informações recolhidas e sistematizadas, argumenta-se que a Análise do Comportamento precisa se voltar mais detidamente para um exame da ansiedade de uma perspectiva cultural, o que se alinha com a proposta de Análise Comportamental da Cultura. A necessidade de novas pesquisas que se proponham a analisar a ansiedade em conjunto com a cultura se justifica, pois, a alta incidência do transtorno no Brasil indica que ansiedade não pode ser elucidada olhando-se apenas para variáveis do âmbito individual, mas que está relacionada também com práticas culturais. Esse entendimento abre espaço para repensar as estratégias de prevenção e enfrentamento desse fenômeno no âmbito de políticas públicas.

\section{REFERÊNCIAS}

ABREU, P. R; PRADA, C. G. Transtorno de ansiedade obsessivo-compulsivo (TOC) e transtorno da personalidade obsessivo-compulsivo (TPOC): Um "diagnóstico" analítico-comportamental. Revista Brasileira de Terapia Comportamental e Cognitiva, v. 6, n. 2, p. 211-220, 2004.

ASSOCIAÇÃO AMERICANA DE PSIQUIATRIA. Manual Diagnóstico e Estatístico de Transtornos Mentais: DSM- 5. 5.ed. Porto Alegre: Artmed, 2014.

ANDERY, M. A. P. A; Comportamento e cultura na perspectiva da análise do comportamento. Perspectivas em Análise do Comportamento, v. 2, n. 2, p. 203-217, 2011.

BANACO, R. A; ZAMIGNANI, D. R; MEYER, S. B. Função do comportamento e do DSM: Terapeutas analítico-comportamentais discutem a psicopatologia. In: TOURINHO, E. Z; DE LUNA, S. V. (Orgs). Análise do comportamento: investigações históricas, conceituais e aplicadas. São Paulo: Roca, 2010. cap, 8, p. 175-191.

BRITTO, I. A. G. S; DUARTE, A. M. M. Transtorno de pânico e agorafobia: um estudo de caso. Revista Brasileira de Terapia Comportamental e Cognitiva, v. 6, n. 2, p. 165-172, 2004.

CUNHA, L. S; BORLOTI, E. B. O efeito de contingências de reforçamento programadas sobre o relato de eventos privados. Revista Brasileira de Terapia Comportamental e Cognitiva, v. 11, n. 2, p. 209-230, 2009. 
GONGORA, M. A. N. Noção de psicopatologia na análise do comportamento. In: COSTA, C. E.; LUZIA, J. C.; SANTANNA, H. H. N. (Orgs.). Primeiros passos em análise do comportamento e cognição. Santo André, SP: ESETec Editores Associados, 2003. p. 93-109.

HENKLAIN, M. H. O; CARMO, J. S. Produção analítico-comportamental sobre ensino-aprendizagem de habilidades matemáticas: Dados representativos de eventos científicos brasileiros. Perspectivas em Análise do Comportamento, v. 2, n. 2, p. 179-191, 2011.

FERREIRA, D. C. et al. A interpretação de cognições e emoções com o conceito de eventos privados e a abordagem analítico-comportamental da ansiedade e da depressão. Perspectivas em Análise do Comportamento, v. 1 , n. 2, p. 70-85, 2010.

MORAES, A. B. A. et al. Análise de Comportamentos de mães que acompanham seus filhos durante tratamento odontológico. Revista Brasileira de Análise do Comportamento, v. 2, n. 2, p. 235-249, 2006.

NEVES, S. M. M. et al. O modelo da equivalência de estímulos na análise de distúrbios de ansiedade: Os efeitos da história experimental e da qualidade de estímulos em sujeitos ansiosos e não-ansiosos. Revista Brasileira de Análise do Comportamento, v. 1, n. 1, p. 57-66, 1999.

NOBILE, G. F. G; GARCIA, V. A; BOLSONI-SILVA, A. T. Análise sequencial dos comportamentos do terapeuta em psicoterapia com universitários com transtorno de ansiedade social. Perspectivas em análise do comportamento, v. 8, n. 1, p. 16-31, 2017.

OLIVEIRA, M. A; DUARTE, A. M. M. Controle de respostas de ansiedade em universitários em situações de exposições orais. Revista Brasileira de Terapia Comportamental e Cognitiva, v. 6, n. 2, p. 183-199, 2004.

ORGANIZAÇÃO MUNDIAL DA SAÚDE. CID-10: Classificação Estatística Internacional de Doenças e Problemas relacionados à Saúde. 10a rev. São Paulo: Universidade de São Paulo, 1997.

PEZZATO, F. A; BRANDÃO, A. S; OSHIRO, C. K. B. Intervenção baseada na psicoterapia analítica functional em um caso de transtorno de pânico com agorafobia. Revista Brasileira de Terapia Comportamental e Cognitiva, v. 14, n. 1, p. 74-84, 2012.

PRADO, R. C. P. Uma leitura analítico-comportamental da psicopatologia. Scientia, v. 1, n 2, p. 192-395, 2013. 
REGIS NETO, D. M. R. et al. Supressão condicionada: Um modelo experimental para o estudo da ansiedade. Perspectivas em Análise do Comportamento, v. 2, n. 1, p. 5-20, 2011.

SANCHEZ, C. N. M.; GOUVEIA JUNIOR, A. Adaptação da EAH para população de surdos falantes de LIBRAS. Revista Brasileira de Terapia Comportamental e Cognitiva, v. 10, n. 2, p. 171-179, 2008.

SANCHEZ, C. N. M.; GOUVEIA JUNIOR, A. O teste da simulação do falar em público não gera ansiedade em adolescentes surdos ou ouvintes. Revista Brasileira de Terapia Comportamental e Cognitiva, v. 13, n. 2, p. 21-32, 2011.

SIDMAN, M. Coerção e suas implicações. Campinas, SP: Editora Livro Pleno, 2009.

SKINNER, B. F. Ciência e comportamento humano. 11. ed. São Paulo: Martins Fontes, 2003.

- Seleção pelas consequências. Revista Brasileira de Terapia Comportamental e Cognitiva, v. 9, n 1, p. 129-137, 2007. (Publicação original de 1981).

Sobre o behaviorismo. 10. ed. São Paulo: Cultrix, 2006.

SOUSA, A. C. A. Transtorno de personalidade borderline sob uma perspectiva analítico-funcional. Revista Brasileira de Terapia Comportamental e Cognitiva, v. 5, n. 2, p. 121-137, 2003.

ULMANN, L. P; KRASNER, L. Defining abnormality. In: psychological approach to abnormal behavior. New Jersey: Prentice-Hall, 1969. cap. 1, p. 9-24.

WORLD HEALTH ORGANIZATION. Depression and Other Common Mental Disorders: Global Health Estimates. Geneva, 2017.

ZAMIGNANI, D. R; BANACO, R. A. Um panorama analítico-comportamental sobre os transtornos de ansiedade. Revista Brasileira de Terapia Comportamental e Cognitiva, v. 7, n 1, p. 77-92, 2005. 